\title{
Ivacaftor treatment in patients with cystic fibrosis and the G551D-CFTR mutation
}

\author{
Isabelle Sermet-Gaudelus
}

\begin{abstract}
Cystic fibrosis (CF) is an autosomal recessive lethal disease caused by mutations in the cystic fibrosis transmembrane conductance regulator (CFTR) gene that encodes for CFTR, an epithelial cell-surface expressed protein responsible for the transport of chloride $\left(\mathrm{Cl}^{-}\right)$. Gating mutations associated with defective conductance can be modulated by CFTR potentiators. Ivacaftor is a CFTR potentiator approved for the treatment of CF patients $>\mathbf{6}$ yrs of age with at least one copy of the G551D-CFTR mutation. Herein, the clinical trial development programme for ivacaftor will be reviewed, including two pivotal studies in adolescents/adults and in children. These studies report sustained improvements in lung function and sweat chloride concentrations, and a reduction in pulmonary exacerbations over a 48-week treatment period. In the era of personalised medicine, ivacaftor offers an effective and well-tolerated treatment for the clinical management of CF patients with the G551D mutation. A long-term, open-label study will report the effects of ivacaftor over a further 48 weeks.
\end{abstract}

KEYWORDS: CFTR mutations, CFTR potentiator, cystic fibrosis, G551D, ivacaftor, personalised medicine

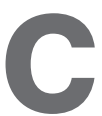
ystic fibrosis (CF) is the most frequent and lethal autosomal disease among the Caucasian population. It is a life-limiting, multisystem disorder that affects the chloride transport system in exocrine tissues. The hallmark is a classic triad of symptoms, most often from infancy or childhood, that associate progressive obstructive lung disease with sputum infected by Staphylococcus aureus or Pseudomonas aeruginosa, exocrine pancreatic insufficiency, and a high sweat chloride level. Mortality is mainly due to progression of lung disease and respiratory insufficiency [1]. CF is due to mutations in the cystic fibrosis transmembrane conductance regulator (CFTR) gene, which encodes for CFTR, an epithelial cell-surface expressed protein that is responsible for transport of chloride $\left(\mathrm{Cl}^{-}\right)$and bicarbonate across the cell membrane. Normal function of this channel is regulated by a cAMPdependent protein kinase-A process [2-4]. Since the discovery of the CFTR gene in 1989, research has been on-going to identify therapeutic strategies to repair the defects in the CFTR gene or CFTR protein [5].

Such mutation-specific therapies aim to silence the deleterious effect of specific mutations or mechanisms of mutations. They address the disease upstream and transcend the strategy of treating secondary events of the disease. The concept of "personalised medicine" has been described previously in the current issue of the European Respiratory Review by ELBORN [6]. Herein, we review a specific example, namely the clinical research programme that led to the approval of ivacaftor; a CFTR modulator for use in CF patients $>6$ yrs of age who have a specific CFTR mutation (G551D).

\section{OVERVIEW OF CFTR AND ITS} \section{RELATIONSHIP TO CF PATHOPHYSIOLOGY} As already discussed by DERICHS [7], volume depletion of the lung airway surface liquid is caused by a reduction in the quantity or activity of CFTR protein in the apical membrane of lung epithelial cells [4]. Accumulation of thickened mucus inevitably leads to impaired mucociliary transport, mucus stasis, airway obstruction and pathogen-induced inflammation. Once obstruction is established, multiple mechanisms perpetuate excessive chronic inflammation, mucus hypersecretion and chronic bacterial infection. This creates a progressive chronic airway disease leading to end-stage lung disease, the primary cause of morbidity and mortality in this disorder [1]. Excessive sweat chloride production is also
AFFILIATION

Centre de Ressources et de Compétences pour la Mucoviscidose, Hopital NeckerEnfants-Malades, INSERM U845, Université René Descartes, Paris, France.

CORRESPONDENCE

I. Sermet-Gaudelus

Centre de Ressources et de Compétences pour la Mucoviscidose

Hopital Necker-Enfants-Malades INSERM U845

Université René Descartes

Paris

France

E-mail: isabelle.sermet@nck.aphp.fr

Received:

Dec 202012

Accepted after revision:

Jan 072013

PROVENANCE

Publication of this peer-reviewed article was supported by Vertex Pharmaceuticals Inc., USA (principal sponsor, European Respiratory Review issue 127). 
characteristic of $\mathrm{CF}$ and in vitro research has found this to be the result of impaired CFTR-mediated reabsorption of chloride in the sweat gland, resulting in the production of salty sweat, a symptom used to diagnose CF [2].

Abnormal function of CFTR can result from either nondelivery of CFTR protein to the apical membrane or from altered ability of the CFTR channel to transport chloride. In the case of absent CFTR, this may result from nonsense mutations leading to defective ribosomal synthesis, a defect which is accessible to therapies over-riding the premature stop signal [8], or from mutations altering intracellular processing, leading to degradation of the misfolded protein; this can be corrected by drugs restoring CFTR trafficking to the cell surface. Such therapies are exemplified by the agent VX-809, which has been shown to rescue channel misfolding caused by the F508del mutation [9]. Mutations associated with defective function of CFTR protein at the apical membrane can affect the conductance of ions (Class IV mutation) or channel gating (Class III mutation). In the latter case, gating mutations can be modulated by CFTR potentiators [3].

\section{THERAPEUTIC TARGETING OF THE G551D MUTATION}

The G551D mutation is the most prevalent gating mutation. It is caused by substitution of the amino acid glycine by aspartate at position 551 in the nucleotide binding domain-1 of the CFTR gene. It abolishes ATP-dependent gating, resulting in an open probability that is approximately 100 -fold lower than that of wild-type channels. It is associated with a severe phenotype, characterised by pulmonary dysfunction and pancreatic insufficiency [10, 11]. G551D, also known as the Celtic mutation, is more common in north-west and central Europe and, in particular, the UK (3.1\%) and Northern Ireland (3.7\%), the Czech Republic (3.8\%), Ireland (5.7\%), Brittany, France $(3.6 \%)$ and Austria (1.2\%). The gene is less common in other parts of Europe [12, 13].

Ivacaftor (VX-770; Vertex Pharmaceuticals, Cambridge, MA, USA) is a potentiator that specifically targets the G551D gating mutation by improving the probability that the mutant channel will open at the cell surface, i.e. increases the channel opening probability and, therefore, the flow of ions transported through the channel. Ivacaftor was discovered using high-throughput screening of cell-culture systems recombinantly expressing G551D-CFTR. The candidate was validated using follow-up in vitro analyses (refer to [7]) before rapidly advancing into clinical trials.

\section{IVACAFTOR PHASE || CLINICAL TRIALS}

In a phase II clinical trial the safety and adverse event profile of ivacaftor was assessed as a primary end-point [14]. Secondary end-points included improvements in CFTR-mediated ion transport (measured by nasal potential difference and sweat chloride concentrations) [15], pulmonary status (measured by forced expiratory volume in $1 \mathrm{~s}$ (FEV1)) and health-related quality of life (measured by the Cystic Fibrosis Questionnairerevised (CFQ-R)). The study was conducted in $10 \mathrm{CF}$ patients who were $\geqslant 18$ yrs and who had at least one copy of the G551D mutation as well as an FEV1 $\geqslant 40 \%$ [16]. This double-blind, placebo-controlled study was conducted in two parts. Part one was a modified cross-over design in which patients were randomly assigned to receive ivacaftor every $12 \mathrm{~h}$ at doses of
25,75 or $150 \mathrm{mg}$ or placebo. The study drug was administered during two 14-day periods, separated by a washout period. Part 2 was a parallel group study involving 19 new subjects who were randomised in a 2:2:1 ratio to receive ivacaftor every $12 \mathrm{~h}$ at a dose of 150 or $250 \mathrm{mg}$ or placebo for 28 consecutive days [14].

The study showed that ivacaftor was well tolerated and the authors recommended follow-up clinical trials to further investigate the drug's efficacy in CF patients with the G551DCFTR mutation. The frequency of adverse events was similar between treatment groups and the most commonly occurring events were fever, cough, nausea, pain and rhinorrhoea. Most importantly, ivacaftor treatment showed within-subject improvements in channel function in both the nasal and the sweat gland epithelium. Moreover, a trend towards normalisation of sweat chloride and nasal potential difference levels was associated with improvements in lung function. In some treated subjects the sweat chloride levels decreased to below the diagnostic cut-off for CF $\left(60 \mathrm{mEq} \cdot \mathrm{L}^{-1}\right)$. This was the first study of a CF therapy to demonstrate normalisation of sweat chloride. Very interestingly, measures of lung function showed significant improvements: the median change from baseline in FEV1 was $8.7 \%$ predicted (range $2.3-31.3, \mathrm{p}=0.008$ ). A clinically important improvement in health-related quality of life (CFQ-R scores) was also reported in ivacaftor treated patients, although this was not significant compared to placebo [14].

This proof-of-concept phase II study therefore confirmed the ability of the drug to restore G551D-dependant CFTR chloride transport. Taken together, these findings supported further clinical investigation of ivacaftor to determine long-term efficacy and safety as a targeted therapy for CF patients with the G551D mutation.

\section{PHASE III CLINICAL TRIALS INVESTIGATE THE EFFICACY OF IVACAFTOR IN ADULTS AND CHILDREN WITH THE G551D-CFTR MUTATION}

Two 48-week randomised, double-blind, placebo-controlled phase III trials were conducted in adolescents/adults (STRIVE (Evaluating the Efficacy and Safety of Treatment with VX-770 in CF patients with G551D Mutation) study) [17] and in children (ENVISION (Evaluation of Efficacy and Safety of VX-770 in children six to eleven years old with CF) study) [18]. At the end of the 48-week study period patients were offered the opportunity to rollover into an optional open-label study (PERSIST; An Open-Label Rollover Study to Evaluate the Safety and Efficacy of VX-770 in Cystic Fibrosis patients), designed to monitor the long-term impact of ivacaftor treatment over 96 weeks. This study is currently ongoing [18]. As the dose of $250 \mathrm{mg}$ twice daily did not show a significant increase in the phase II study when compared with $150 \mathrm{mg}$ twice daily, the latter dose was used for the phase III studies.

To participate in the STRIVE study, CF subjects had to be aged $\geqslant 12$ yrs and possess at least one copy of the G551D mutation. Patients were also required to have an FEV1 of $40-90 \%$ pred of normal [17]. The ENVISION study, conducted in children aged 6-11 yrs, had similar recruitment criteria, with the exception of a pre-specified required for FEV1 measurements of $40-105 \%$ pred of normal [18]. This was to address the smaller lung 


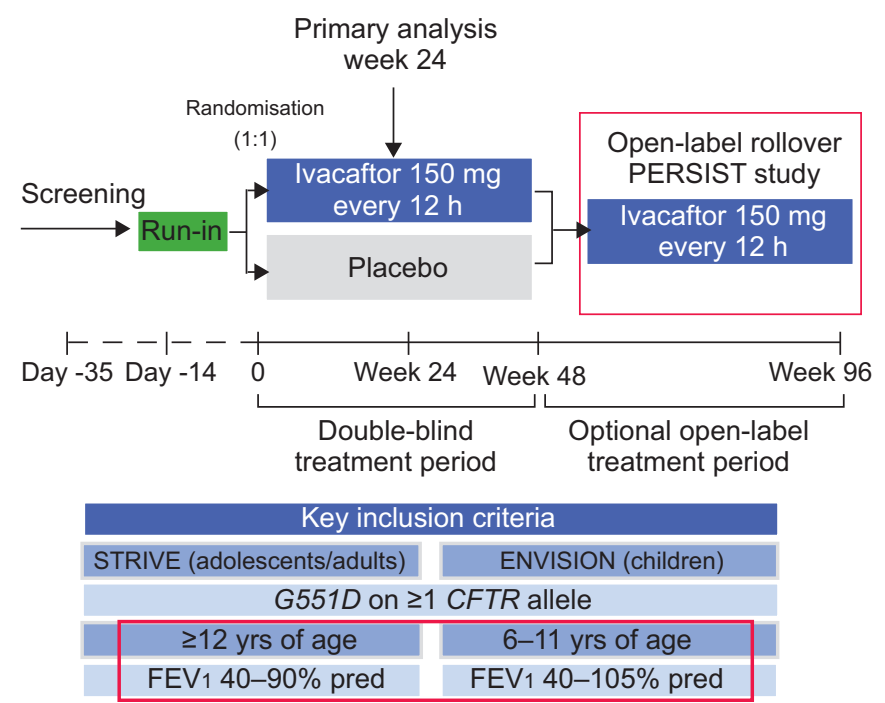

FIGURE 1. Design of adult and paediatric studies investigating the efficacy and tolerability of ivacaftor $[16,17]$. PERSIST: An Open-Label Rollover Study to Evaluate the Safety and Efficacy of VX-770 in Cystic Fibrosis Patients; STRIVE: Evaluating the Efficacy and Safety of Treatment with VX-770 in CF patients with G551D Mutations; ENVISION: Evaluation of Efficacy and Safety of VX-770 in children six to eleven years old with CF; CFTR: cystic fibrosis transmembrane conductance regulator; FEV 1 : forced expiratory volume in $1 \mathrm{~s}$; \% pred: \% predicted.

capacity of paediatric subjects and due to the fact that lung disease is less severe in the paediatric population.

The study design for both STRIVE and ENVISION was similar (fig. 1). Following a 14-day run-in period, patients were randomised to receive ivacaftor $150 \mathrm{mg}$ twice daily (83 adults, 26 children) or placebo (78 adults, 26 children) for the 48 -week study period. The primary efficacy end-point in both STRIVE and ENVISION was the absolute change from baseline to week 24 in FEV1 \% pred. Secondary end-points included the change in FEV1 \% pred from baseline to week 48 , time-to-first pulmonary exacerbation and patient-reported respiratory symptoms through to weeks 24 and 48. Other secondary end-points included change in weight and sweat chloride concentration (a measure of CFTR channel function) from baseline through to weeks 24 and 48. Upon completion of the 48-week treatment period, patients were eligible to rollover into the PERSIST study to receive open-label ivacaftor for an additional 96 weeks. In the STRIVE study, baseline characteristics were comparable between treatment groups in both studies.

\section{Lung function and clinical efficacy}

In the STRIVE study, a significant improvement in lung function was observed due to ivacaftor treatment. At week 24 the FEV1 \% pred showed a statistically significant treatment effect of $10.6 \%$ points (10.4\% points with ivacaftor, $-0.2 \%$ points with placebo; $\mathrm{p}<0.001$ ) (fig. 2). Moreover, this ivacaftor-dependent improvement was noted as early as 15 days after the study commenced. This significant treatment effect was maintained throughout the study, with a treatment effect through to week 48 of $10.5 \%$ points greater with ivacaftor than with placebo $(p<0.001)$ [17].

Similarly the ENVISION study, conducted in paediatric patients, reported a mean absolute change from baseline in

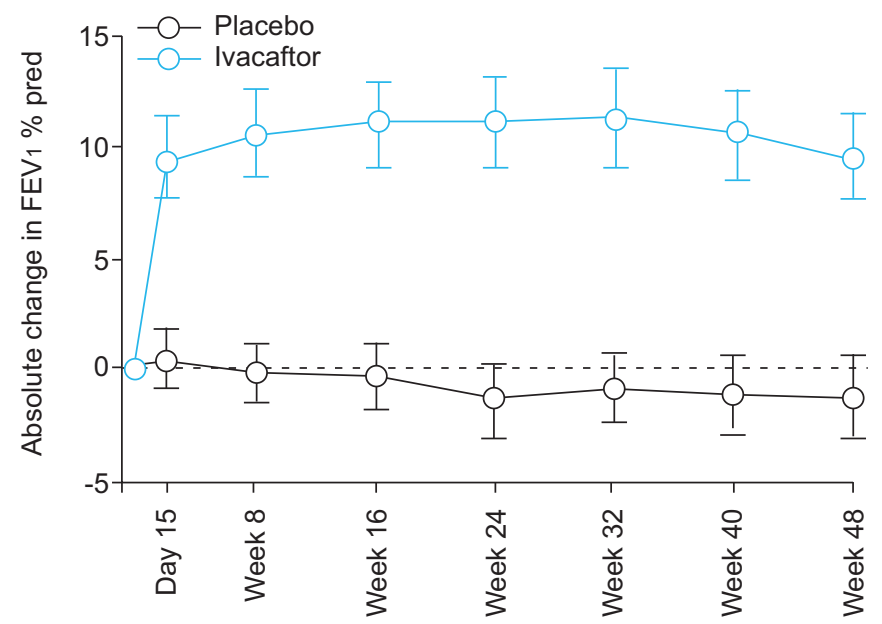

FIGURE 2. Results from the the STRIVE study of forced expiratory volume in $1 \mathrm{~s}$ (FEV 1) absolute change from baseline. Treatment effect from baseline to week $24:+10.6 \%, p<0.0001$. Treatment effect from baseline to week $48:+10.5 \%$, $p=0.0001$. Data are presented as mean $(95 \% \mathrm{Cl}) . \%$ pred: \% prediced.

FEV1 of $12.5 \%$ pred $(\mathrm{p}<0.0001)$ through to 24 weeks and $10.0 \%$ $(p=0.0006)$ through to 48 weeks [18].

In STRIVE, the absolute change in FEV1 \% pred measured at each study visit through to week 24 , showed that nearly $75 \%$ of ivacaftor-treated adults had a mean improvement of $5 \%$ points. Interestingly, improvements in patients with poor pulmonary function were similar to those in patients with only mild functional impairment. The responses in younger $\mathrm{CF}$ patients with mild disease were particularly striking, even for those with normal FEV1 \% pred. However, a degree of caution should be exercised when interpreting subgroup analyses containing relatively small participant numbers [17].

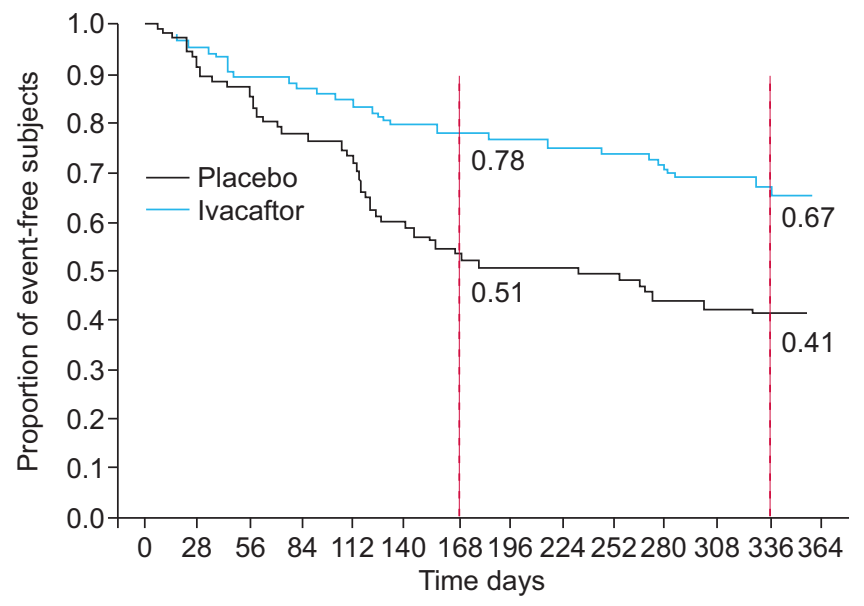

FIGURE 3. Time to first pulmonary exacerbation in adults treated with ivacaftor or placebo according to the modified Fuch's criteria. The dotted lines represent weeks 24 (168 days) and 48 (336 days), respectively. Week 24: hazard ratio $=0.40$, $p=0.0016$. Week 48: hazard ratio $=+10.5 \%, p=0.0001$. 
The rate of pulmonary exacerbations was also significantly improved. At week 48 in the STRIVE study, $67 \%$ of subjects in the ivacaftor group were free from pulmonary exacerbations compared to $41 \%$ in the placebo group. This corresponded to a $55 \%$ reduction in the risk of pulmonary exacerbation $(\mathrm{p}=0.001)$ (fig. 3). The mean \pm SD total number of days of hospitalisation for pulmonary exacerbations per patient (normalised to a 48week period) was $3.9 \pm 13.6$ in the ivacaftor group compared to $4.2 \pm 8.7$ in the placebo group $(p=0.03)$ [17].

These results support the hypothesis that improvement in CFTR function leads to enhanced airway clearance. Nevertheless, the cellular mechanisms that facilitate improvements in lung function are not yet fully understood [17].

\section{Influence of ivacaftor on patient-reported outcomes}

Patient-reported respiratory symptoms were assessed using the respiratory domain of the CFQ-R, which has been specifically validated for use in CF. Responses are scored on a 100-point scale, with higher numbers indicating a lower effect of symptoms on the patient's quality of life and a change of four points considered to be a minimal clinically important difference [20]. Findings showed an improvement in CFQ-R scores, indicating a reduction in respiratory symptoms. From baseline to week 48 , the scores increased by 5.9 points in the ivacaftor group, compared with a decrease of 2.7 points in the placebo group (treatment effect 8.6 points; $\mathrm{p}<0.001$ ).

\section{Nutritional status}

Weight gain occurred at a significantly greater extent among patients treated with ivacaftor. In the STRIVE study, by week 48 subjects in the ivacaftor group had gained a mean of $3.1 \mathrm{~kg}$ compared to a mean gain of $0.4 \mathrm{~kg}$ in the placebo group (treatment effect $2.7 \mathrm{~kg} ; \mathrm{p}<0.001$ ), with weight gain appearing to plateau at 16 weeks. This may indicate that patients had reached their ideal weight or other factors prevented them from gaining further weight [17]. A significant increase in weight was also noted with ivacaftor in the ENVISION study.

\section{Sweat chloride concentrations}

Sweat chloride levels in treated subjects demonstrated a rapid and sustained response. There was a mean reduction in measured sweat chloride levels of $48.1 \mathrm{mmol} \cdot \mathrm{L}^{-1} \quad(\mathrm{p}<0.001)$ compared to placebo; this effect was observed at day 15 and was maintained through to week 48 [17]. Furthermore, some sweat chloride levels in ivacaftor treated patients were lowered to below the diagnostic threshold of CF $\left(60 \mathrm{mEq} \cdot \mathrm{L}^{-1}\right)$. Similarly, in the ENVISION study ivacaftor led to a significant reduction in sweat chloride through to week 48 (treatment difference $\left.-53.5 \mathrm{mmol} \cdot \mathrm{L}^{-1} ; \mathrm{p}<0.0001\right)$. However, no direct correlation was observed between sweat chloride concentrations and pulmonary manifestation as measured by FEV1 (fig. 4). This suggests that measurement of sweat chloride levels may not be sufficient to provide reassurance of the clinical efficacy of treatment. Nevertheless, although the findings suggest that sweat chloride concentrations cannot predict treatment efficacy, the "sweat test" may still be useful in determining response to treatment [7].

\section{Safety and tolerance of ivacaftor}

Adverse event data from both the STRIVE and ENVISION studies were pooled to obtain insight into the safety profile of

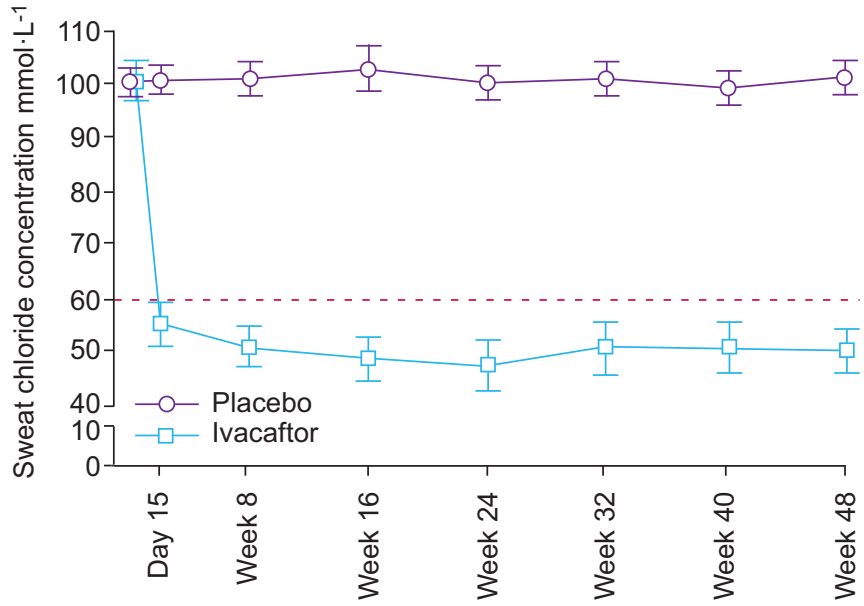

FIGURE 4. Sweat chloride concentrations from baseline to week 48 of the study. Treatment effect from baseline to week 24: $-47.9 \mathrm{mmol} \cdot \mathrm{L}^{-1}, \mathrm{p}<0.0001$. Treatment effect from baseline to week 48 : $-48.1 \mathrm{mmol} \cdot \mathrm{L}^{-1}, \mathrm{p}<0.0001$. Data are presented as mean $(95 \% \mathrm{Cl})$. $\cdots \cdots \cdots$. diagnostic threshold.

ivacaftor. The incidence of adverse events was similar in both ivacaftor treatment groups through to week 48 (table 1). Ivacaftor treated subjects had a higher incidence of adverse events leading to interruption of the study drug (13\% versus $6 \%$ ). The only adverse event that led to the discontinuation of ivacaftor was an increase in hepatic enzymes; nevertheless, all subjects were able to resume taking the study drug and to complete the trial. Most commonly reported adverse events $(\geqslant 8 \%$ of the study population) among ivacaftor treated patients included headache, upper respiratory tract infection, nasal congestion, rash and dizziness (table 1). These events were not significantly different compared with placebo and none of them led to study discontinuation.

\section{Long-term impact of ivacaftor treatment}

Interim results from the first 12 weeks of PERSIST, including the 144 adults who rolled over from STRIVE, were published

\section{TABLE 1 Adverse events occurring in invacaftor and placebo-treated patients: pooled data from adults (STRIVE trial) and children (ENVISION trial)}

\begin{tabular}{lcc} 
Adverse event $^{\#}$ & Ivacaftor $^{\boldsymbol{T}}$ & Placebo $^{+}$ \\
\hline Headache & $26(24)$ & $17(16)$ \\
Oropharyngeal pain & $24(22)$ & $19(18)$ \\
Upper respiratory tract infection & $24(22)$ & $14(14)$ \\
Nasal congestion & $22(20)$ & $16(15)$ \\
Abdominal pain & $17(16)$ & $13(13)$ \\
Nasopharyngitis & $16(15)$ & $12(12)$ \\
Diarrohea & $14(13)$ & $10(10)$ \\
Rash & $14(13)$ & $7(7)$ \\
Nausea & $13(12)$ & $11(11)$ \\
Dizziness & $10(9)$ & $1(1)$ \\
\hline
\end{tabular}

Data are presented as $n(\%)$. No significant signals were identified through to week 48. ${ }^{*}$ : occurring more commonly $(\geqslant 8 \%)$ in ivacaftor-treated subjects: : $n=109 ;{ }^{+}: n=104$ 
recently [19]. This preliminary analysis observed that improvements in FEV1 reported during STRIVE were sustained for an additional 12 weeks beyond the initial 48-week study period (treatment difference $9.4 \%$ points). An improvement in FEV1 was also noted for those who switched from placebo to ivacaftor at the beginning of PERSIST $(n=67)$. The magnitude of this improvement at 15 days after the start of active treatment was similar to that reported in STRIVE, thus, further supporting the beneficial effect of ivacaftor [19].

Preliminary results from PERSIST also strengthened evidence that ivacaftor has a beneficial effect on CF patients with severe disease. Eight patients in the group who switched from placebo to ivacaftor at the start of PERSIST had an FEV1 $<40 \%$ pred (indicating severe disease). In this subgroup, the mean \pm FEV $1 \%$ pred on day 1 of STRIVE was $34.5 \pm 3.7 \%$. This improved to a mean \pm SD absolute change of $10.8 \pm 8.7 \%$ at day 15 and a mean \pm SD absolute change of $13.0 \pm 10.5 \%$ at week 12 [19]. Similarly, the reductions in the number and duration of pulmonary exacerbations of patients initially in the placebo group after entering the open label study were similar to those reductions observed in subjects in the placebo-controlled ivacaftor study.

\section{IMPACT OF IVACAFTOR ON CF PATIENTS WITH THE F508DEL-CFTR MUTATION}

Ivacaftor has also been studied in patients homozygous for the F508del mutation, the most frequent mutation in the Caucasian population. This is because this mutation not only has trafficking defects but also affects the function of the protein. Indeed, even the small amount of F508del-CFTR protein that reaches the cell surface opens less frequently and is retrieved from the membrane much faster than the wild-type protein. Such a defect might, therefore, be accessible to potentiators, which improve the functional activity of the residual protein.

In vitro studies have shown that ivacaftor also acts as a "modest" potentiator to the small amount of F508del-CFTR channels that are trafficked to the cell surface [21]. In addition, human bronchial primary cells derived from lung biopsies of F508del-CFTR homozygous patients demonstrated a limited response to potentiation of CFTR [21-23].

This pre-clinical evidence provided the basis for DISCOVER, a two-part, phase II randomised, double-blind parallel group study to investigate the effects of ivacaftor in patients homozygous for the F508del-CFTR mutation [24]. The study involved oral administration of $150 \mathrm{mg}$ of ivacaftor or placebo every $12 \mathrm{~h}$ for 16 weeks, followed by an open-label 96-week extension for suitable subjects. The primary efficacy end-point was the absolute change in FEV1 \% pred from baseline through to week 16 in a total of 140 randomised subjects (112 ivacaftor and 28 placebo). Study results showed that ivacaftor was not associated with significant improvements in FEV1 or other clinical endpoints including CFQ-R or the rate of pulmonary exacerbations. Although a small reduction in sweat chloride concentration was noted during the first part of the study, this was not observed in the open-label extension. The study concluded that ivacaftor would not be beneficial for those who are homozygous for the F508del-CFTR mutation [24]. Nevertheless, a clinical study is underway to assess the efficacy and safety of VX-809 (a CFTR corrector) used alone and in combination with ivacaftor (a CFTR potentiator) to treat $\mathrm{CF}$ patients homozygous or heterozygous for the F508del-CFTR mutation (clinicaltrials.gov NCT01225211). If positive, the outcome of this trial could benefit the vast majority of CF patients worldwide.

\section{CONCLUSION}

Clinical development of ivacaftor is an outstanding example of how personalised medicine can revolutionise medicine. Following identification of ivacaftor using high throughput screening, early pre-clinical research validated this small molecule as a promising therapeutic option for $\mathrm{CF}$ patients with the G551D-CFTR mutation. Two pivotal clinical trials in adult and paediatric CF patients with the G551D mutation demonstrated rapid, dramatic and sustained improvements in FEV1, self-reported outcomes of respiratory symptoms, nutritional status and reduced pulmonary exacerbations by $>50 \%$. Efficacy outcomes in children were consistent with those in the older population despite milder disease at baseline, suggesting that early disease might be reversible or at least preventable. A significant change in sweat chloride levels mirrored improvements in lung function, as a proof-of-concept of the drug mechanism of action. There were no important safety concerns for $>48$ weeks administration. Initial analysis of an ongoing longer term open-label study suggests that the efficacy of ivacaftor will be maintained in the long-term. In contrast, ivacaftor did not display any clinical efficacy or bring about a significant reduction in sweat chloride levels in patients with the F508del mutation.

Such studies validate the knowledge that mutation-specific therapy can ultimately make a difference in the clinical setting. Such innovative multisystem treatment of an underlying defect demonstrates how future therapeutic choices could be realistically driven by personalised genetic information. This represents an important milestone in $\mathrm{CF}$ and will pave the way for the development of other disease-modifying drugs in respiratory medicine and beyond.

\section{STATEMENT OF INTEREST}

I. Sermet-Gaudelus has received speaker honorarium and reimbursement for attending a symposium from Vertex Inc.

\section{ACKNOWLEDGEMENTS}

I would like to thank Facilitate Ltd (Brighton, UK), funded by Vertex Pharmaceuticals Inc., USA, for editorial assistance in the production of this manuscript.

\section{REFERENCES}

1 Flume PA, Van Devanter DR. State of progress in treating cystic fibrosis respiratory disease. BMC Med 2012; 10: 88.

2 Rowe SM, Miller S, Sorscher EJ. Cystic fibrosis. N Engl J Med 2005; 352: 1992-2001.

3 Rogan MP, Stoltz DA, Hornick DB. Cystic fibrosis transmembrane conductance regulator intracellular processing, trafficking, and opportunities for mutation-specific treatment. Chest 2011; 139: 1480-1490.

4 Boucher RC. New concepts of the pathogenesis of cystic fibrosis lung disease. Eur Respir J 2004; 23: 146-158.

5 De Boeck K, Kent L, Davies J, et al. CFTR biomarkers: time for promotion to surrogate end-point. Eur Respir J 2013; 41: 203-216.

6 Elborn JS. Personalised medicine for cystic fibrosis: treating the basic defect. Eur Respir Rev 2013; 22: 3-5. 
7 Derichs N. Targeting a genetic defect: cystic fibrosis transmembrane conductance regulator modulators in cystic fibrosis. Eur Respir Rev 2013; 22: 58-65.

8 Pettit RS. Cystic fibrosis transmembrane conductance regulatormodifying medications: the future of cystic fibrosis treatment. Ann Pharmacother 2012; 46: 1065-1075.

9 Van Goor F, Hadida S, Grootenhuis PD, et al. Correction of the F508del-CFTR protein processing defect in vitro by the investigational drug VX-809. Proc Natl Acad Sci USA 2011; 108: 18843-18848.

10 Bompadre SG, Sohma Y, Li M, et al. G551D and G1349D, two CFassociated mutations in the signature sequences of CFTR, exhibit distinct gating defects. J Gen Physiol 2007; 129: 285-298.

$11 \mathrm{Yu} \mathrm{H}$, Burton $\mathrm{B}$, Huang $\mathrm{CJ}$, et al. Ivacaftor potentiation of multiple CFTR channels with gating mutations. J Cyst Fibros 2012; 11: 237-245.

12 Bobadilla JL, Macek M Jr, Fine JP, et al. Cystic fibrosis: a worldwide analysis of CFTR mutations - correlation with incidence data and application to screening. Human Mutat 2002; 19: 575-606.

13 Estivill X, Bancells C, Ramos C. Geographic distribution and regional origin of 272 cystic fibrosis mutations in European populations. The Biomed CF Mutation Analysis Consortium. Human Mutat 1997; 10: 135-154.

14 Accurso FJ, Rowe SM, Clancy JP, et al. Effect of VX-770 in persons with cystic fibrosis and the G551D-CFTR mutation. N Engl J Med 2010; 363: 1991-2003.

15 Bronsveld I, Vermeulen F, Sands D, et al. Influence of perfusate temperature on nasal potential difference. Eur Respir J 2012 [Epub ahead of print DOI: 10.1183/09031936.00097712].
16 Knudson RJ, Lebowitz MD, Holberg CJ, et al. Changes in the normal maximal expiratory flow-volume curve with growth and aging. Am Rev Respir Dis 1983; 127: 725-734.

17 Ramsey BW, Davies J, McElvaney NG, et al. A CFTR potentiator in patients with cystic fibrosis and the G551D mutation. N Engl J Med 2011; 365: 1663-1672.

18 Davies JCLH, Yen K, Ahrens R. Ivacaftor in subjects 6 to 11 years of age with cystic fibrosis and the G551D-CFTR mutation. J Cyst Fibros 2012; 11: Suppl. 1, S13.

19 McKone ELH, Yen K, Davies JC. Long-term safety and efficacy of ivacaftor in subjects with cystic fibrosis who have the G551DCFTR Mutation. J Cyst Fibros 2012; 11: Suppl. 1, S13.

20 Quittner AL, Buu A, Messer MA, et al. Development and validation of the Cystic Fibrosis Questionnaire in the United States: a health-related quality-of-life measure for cystic fibrosis. Chest 2005; 128: 2347-2354.

21 Van Goor F, Straley KS, Cao D, et al. Rescue of DeltaF508-CFTR trafficking and gating in human cystic fibrosis airway primary cultures by small molecules. Am J Physiol Lung Cell Mol Physiol 2006; 290: L1117-L1130.

22 Bronsveld I, Mekus F, Bijman J, et al. Chloride conductance and genetic background modulate the cystic fibrosis phenotype of Delta F508 homozygous twins and siblings. J Clin Invest 2001; 108: 1705-1715.

23 Veeze HJ, Halley DJ, Bijman J, et al. Determinants of mild clinical symptoms in cystic fibrosis patients. Residual chloride secretion measured in rectal biopsies in relation to the genotype. J Clin Invest 1994; 93: 461-466.

24 Flume PA, Liou TG, Borowitz DS, et al. Ivacaftor in subjects with cystic fibrosis who are homozygous for the F508del-CFTR mutation. Chest 2012; 142: 718-724. 